International Journal of Current Microbiology and Applied Sciences

ISSN: 2319-7706 Volume 6 Number 3 (2017) pp. 2196-2204

Journal homepage: http://www.ijcmas.com

Original Research Article

https://doi.org/10.20546/ijcmas.2017.603.250

\title{
Bio Efficacy of Botanical Insecticides against Defoliators Pests on Soybean
}

\author{
Toshima Kushram ${ }^{1}$, Y.K. Yadu ${ }^{1}$, M.K. Sahu ${ }^{2}$, A.K. Kulmitra ${ }^{3}$ and R. Kumar ${ }^{4}$ \\ ${ }^{1}$ Department of Entomology, COA, IGKV, Raipur, Chhattisgarh (492012), India \\ ${ }^{2}$ Department of Floriculture and Landscape Architecture, COA, IGKV, Raipur, \\ Chhattisgarh (492012), India \\ ${ }^{3}$ Department of Plant Pathology, COA, IGKV, Raipur, Chhattisgarh (492012), India \\ ${ }^{4}$ Department of Genetics and Plant breeding, COA, IGKV, Raipur, Chhattisgarh (492012), India \\ *Corresponding author
}

\section{A B S T R A C T}

Keywords

Botanical insecticides, Defoliator, Soybean.

Article Info

Accepted: 20 February 2017 Available Online: 10 March 2017
The present study was conducted during kharif, 2015 at Indira Gandhi Krishi Vishwavidyalaya, Raipur (C.G.). The experiment was laid out in Randomized Block Design (RBD) with nine treatments and three replications. Defoliators are the most damaging insect pest of soybean. In the evaluation of plant products against defoliators of soybean garlic+green chilli@8.75 kg/ha was most effective against defoliators $S$. litura and $C$. acuta after first spray having $0.43,0.52$ and in second spray having 0.43 and 0.37 larva/mrl with benefit cost ratio of 8.02 .

\section{Introduction}

Soybean, Glycine $\max (\mathrm{L}$.$) Merill ranks first$ in the world for production of edible oil. India ranks third in world in respect of area and fifth in terms of production. It is an annual crop, fairly easy to grow, that produces more protein and oil per unit of land than almost any other crop. It is a versatile food plant that, used in its various forms, is capable of supplying most nutrients. It can substitute for meat and to some extent for milk. It is a crop capable of reducing protein malnutrition. In addition, soybeans are a source of high value animal feed.

The defoliators, S. litura and $C$. acuta are serious pest on soybean. The full grown caterpillars are most voracious feeders and cause extensive damage by defoliation. Because of excessive and indiscriminate use of pesticide several problems like development of resistance in targeted species, resurgence of secondary pest, elimination of natural enemies and wild life, contamination of soil, water and food chain and wholesome pollution of environment (Asoken et al., 2000).

Botanical pesticides are the important alternatives to minimize or replace the use of synthetic pesticides as they possess an array of properties including toxicity to the pest, repellency, antifeedance, insect growth 
regulatory activities against pests of agricultural importance (Prakash and Rao, 1989, 2003).

The current trends of modern society towards'green consumerism' desiring fewer synthetic ingredients in food may favour plant-based products which are generally recognized as safe in ecofriendly management of plant pests as botanical pesticides (Isman et al., 2006).

\section{Materials and Methods}

A field experiment was laid out in randomized block design with nine treatments, Neem oil (2\%), Karanj oi (2\%), NSKE (5\%), Karanj seed extract (2.5\%), Garlic+green chilli $(8.75 \mathrm{~kg})$, green chilli (9kg), Red chilli (9kg), Triazophos 40 EC $(750 \mathrm{ml})$ and including untreated control replicated three times. The crop was sown on $15^{\text {th }}$ July, 2015 in plot size of $5 \mathrm{~m} \times 4 \mathrm{~m}$ at Indira Gandhi Krishi Vishwavidyalaya, Raipur (C.G.).

In this experiment, observations on the efficacy of treatments were recorded one day before the spray and after 3, 7, and 15 days of first and second spraying of plant products.

\section{Defoliator pests}

Tobacco caterpillar and green semilooper were observed as the major defoliator pests. The observations of these pests were recorded by counting the no. of larvae per meter row length.

\section{Results and Discussion}

Over all mean population of S.litura after first spray

Mean larval population during first spray indicated that chemical insecticide i.e triazophos @ $750 \mathrm{ml} / \mathrm{ha}$ recorded the minimum population of 0.37 larva per meter row length and among the different plant products, garlic+green chilli @ 8.75 kg/ha recorded minimum larval population with 0.43 larva/mrl, followed by NSKE @ 5\% (0.50), Karanj seed extract @ 2.5\% (0.57), Karanj oil @2\%(0.63), Neem oil @2\% (0.70), green chilli@9kg/ha (0.77) and it was maximum in red chilli $9 \mathrm{~kg} / \mathrm{ha}(0.82)$ larva/mrl.

\section{Percent reduction of S.litura population over control after first spray}

Percent reduction of S.litura population was higher in triazophos@ $0750 \mathrm{ml} / \mathrm{ha}(68.64 \%)$ treated crop and among the plant products, it was maximum in garlic+green chilli @ 8.75 $\mathrm{kg} / \mathrm{ha}(63.56 \%)$ treated crop which was followed by NSKE @ 5\% (57.63\%), Karanj seed extract@2.5\% (51.69\%), Karanj oil @ $2 \%$ (46.61\%), Neem oil @ 2\% (40.67\%), green chilli@9kg/ha (34.75\%) and lowest in red chilli@9kg/ha treated plots and was recorded only 30.51 percent reduction in insect population.

\section{Over all mean population of S.litura after second spray}

Over all mean population of S.litura Mean larval population during second spray indicated that chemical insecticide i.e. triazophos @ $750 \mathrm{ml} / \mathrm{ha}$ recorded the minimum population of 0.37 larva per meter row length and among the different plant products, garlic+green chilli @ $8.75 \mathrm{~kg} / \mathrm{ha}$ recorded minimum larval population with 0.43 larva $/ \mathrm{mrl}$, followed by NSKE @ 5\% (0.48), Karanj seed extract @ 2.5\% (0.57), green chilli @ 9kg/ha (0.60), Neem oil @ 2\% (0.58), Karanj oil @ 2\% (0.58) and maximum population in red chilli @ $9 \mathrm{~kg} / \mathrm{ha}$ (0.72larva/mrl). 
Table.1 Efficacy of plant products against Tobacco caterpillar (S. litura) in soybean during Kharif, 2015 after first spray

\begin{tabular}{|c|c|c|c|c|c|c|c|c|c|c|}
\hline \multicolumn{11}{|c|}{ Mean population of tobacco caterpillar/mrl } \\
\hline & & \multicolumn{8}{|c|}{ Post treatment observation } & \multirow{2}{*}{$\begin{array}{c}\text { Percent } \\
\text { reduction of } \\
\text { insect population } \\
\text { over control }\end{array}$} \\
\hline & Treatments & Dose & $\begin{array}{c}\text { Quantit } \\
\text { y /ha }\end{array}$ & $\begin{array}{l}\text { Pre treat. } \\
\text { Obs. }\end{array}$ & 1 DAS & 3 DAS & 7 DAS & 15 DAS & Mean & \\
\hline $\mathrm{T} 1$ & Neem oil & $2 \%$ & $101 \mathrm{t}$ & $\begin{array}{c}0.80 \\
(1.14)^{*}\end{array}$ & $\begin{array}{c}0.73 \\
(1.10)\end{array}$ & $\begin{array}{c}0.60 \\
(1.05)\end{array}$ & $\begin{array}{c}0.67 \\
(1.08)\end{array}$ & $\begin{array}{c}0.80 \\
(1.14)\end{array}$ & $\begin{array}{c}0.70 \\
(1.09)\end{array}$ & 40.67 \\
\hline $\mathrm{T} 2$ & Karanj oil & $2 \%$ & $101 \mathrm{t}$ & $\begin{array}{c}0.73 \\
(1.11)\end{array}$ & $\begin{array}{c}0.67 \\
(1.08)\end{array}$ & $\begin{array}{c}0.53 \\
(1.01)\end{array}$ & $\begin{array}{c}0.60 \\
(1.05)\end{array}$ & $\begin{array}{c}0.73 \\
(1.11)\end{array}$ & $\begin{array}{c}0.63 \\
(1.06)\end{array}$ & 46.61 \\
\hline T 3 & NSKE & $5 \%$ & $251 \mathrm{t}$ & $\begin{array}{c}0.67 \\
(1.08)\end{array}$ & $\begin{array}{c}0.53 \\
(1.02)\end{array}$ & $\begin{array}{c}0.40 \\
(0.95)\end{array}$ & $\begin{array}{c}0.47 \\
(0.98)\end{array}$ & $\begin{array}{c}0.60 \\
(1.05)\end{array}$ & $\begin{array}{c}0.50 \\
(1.00)\end{array}$ & 57.63 \\
\hline $\mathrm{T} 4$ & $\begin{array}{l}\text { Karanj seed } \\
\text { extract }\end{array}$ & $2.5 \%$ & $12.51 \mathrm{t}$ & $\begin{array}{c}0.73 \\
(1.11)\end{array}$ & $\begin{array}{c}0.60 \\
(1.05)\end{array}$ & $\begin{array}{c}0.47 \\
(0.98)\end{array}$ & $\begin{array}{c}0.53 \\
(1.01)\end{array}$ & $\begin{array}{c}0.67 \\
(1.08)\end{array}$ & $\begin{array}{c}0.57 \\
(1.03)\end{array}$ & 51.69 \\
\hline T 5 & $\begin{array}{l}\text { Garlic+green } \\
\text { chilli }\end{array}$ & $8.75 \mathrm{~kg}$ & $8.75 \mathrm{~kg}$ & $\begin{array}{c}0.60 \\
(1.05)\end{array}$ & $\begin{array}{c}0.47 \\
(0.98)\end{array}$ & $\begin{array}{c}0.33 \\
(0.91)\end{array}$ & $\begin{array}{c}0.40 \\
(0.94)\end{array}$ & $\begin{array}{c}0.53 \\
(1.01)\end{array}$ & $\begin{array}{c}0.43 \\
(0.96)\end{array}$ & 63.56 \\
\hline Т 6 & Green chilli & $9 \mathrm{~kg}$ & $9 \mathrm{~kg}$ & $\begin{array}{c}0.73 \\
(1.11) \\
\end{array}$ & $\begin{array}{c}0.80 \\
(1.14) \\
\end{array}$ & $\begin{array}{c}0.67 \\
(1.08) \\
\end{array}$ & $\begin{array}{c}0.73 \\
(1.11) \\
\end{array}$ & $\begin{array}{c}0.87 \\
(1.17) \\
\end{array}$ & $\begin{array}{c}0.77 \\
(1.12) \\
\end{array}$ & 34.75 \\
\hline $\mathrm{T} 7$ & Red chilli & $9 \mathrm{~kg}$ & $9 \mathrm{~kg}$ & $\begin{array}{c}0.80 \\
(1.14)\end{array}$ & $\begin{array}{c}0.80 \\
(1.14)\end{array}$ & $\begin{array}{c}0.73 \\
(1.11)\end{array}$ & $\begin{array}{c}0.80 \\
(1.13)\end{array}$ & $\begin{array}{c}0.93 \\
(1.19)\end{array}$ & $\begin{array}{c}0.82 \\
(1.14)\end{array}$ & 30.51 \\
\hline T 8 & $\begin{array}{l}\text { Triazophos } \\
40 \text { EC }\end{array}$ & $750 \mathrm{ml}$ & $750 \mathrm{ml}$ & $\begin{array}{c}0.53 \\
(1.02)\end{array}$ & $\begin{array}{c}0.40 \\
(0.95)\end{array}$ & $\begin{array}{c}0.27 \\
(0.87)\end{array}$ & $\begin{array}{c}0.33 \\
(0.91)\end{array}$ & $\begin{array}{c}0.47 \\
(0.98)\end{array}$ & $\begin{array}{c}0.37 \\
(0.93)\end{array}$ & 68.64 \\
\hline Т 9 & Control & - & - & $\begin{array}{c}0.87 \\
(1.17)\end{array}$ & $\begin{array}{c}1.07 \\
(1.25)\end{array}$ & $\begin{array}{c}1.13 \\
(1.28)\end{array}$ & $\begin{array}{c}1.20 \\
(1.30)\end{array}$ & $\begin{array}{c}1.33 \\
(1.35)\end{array}$ & $\begin{array}{c}1.18 \\
(1.30)\end{array}$ & - \\
\hline & C.D. at $5 \%$ & & & NS & 0.12 & 0.10 & 0.17 & 0.11 & - & - \\
\hline
\end{tabular}


Table.2 Efficacy of plant products against Tobacco caterpillar (S. litura) in soybean during Kharif, 2015 after second spray

\begin{tabular}{|c|c|c|c|c|c|c|c|c|c|c|}
\hline \multicolumn{11}{|c|}{ Mean population of tobacco caterpillar/mrl } \\
\hline & & \multicolumn{8}{|c|}{ Post treatment observation } & \multirow{2}{*}{$\begin{array}{c}\text { Percent } \\
\text { reduction of insect } \\
\text { population over } \\
\text { control }\end{array}$} \\
\hline & Treatments & Dose & $\begin{array}{c}\text { Quantit } \\
\text { y /ha }\end{array}$ & $\begin{array}{l}\text { Pre treat. } \\
\text { Obs. }\end{array}$ & 1 DAS & 3 DAS & 7 DAS & 15 DAS & Mean & \\
\hline $\mathrm{T} 1$ & Neem oil & $2 \%$ & $101 \mathrm{t}$ & $\begin{array}{c}0.60 \\
(1.05)^{*}\end{array}$ & $\begin{array}{c}0.53 \\
(1.02)\end{array}$ & $\begin{array}{c}0.47 \\
(0.98)\end{array}$ & $\begin{array}{c}0.60 \\
(1.05)\end{array}$ & $\begin{array}{c}0.73 \\
(1.11)\end{array}$ & $\begin{array}{c}0.58 \\
(1.04)\end{array}$ & 47.27 \\
\hline T 2 & Karanj oil & $2 \%$ & $101 \mathrm{t}$ & $\begin{array}{c}0.53 \\
(1.01) \\
\end{array}$ & $\begin{array}{c}0.47 \\
(0.98) \\
\end{array}$ & $\begin{array}{c}0.40 \\
(0.95) \\
\end{array}$ & $\begin{array}{c}0.67 \\
(1.08) \\
\end{array}$ & $\begin{array}{c}0.80 \\
(1.14) \\
\end{array}$ & $\begin{array}{c}0.58 \\
(1.04) \\
\end{array}$ & 47.27 \\
\hline T 3 & NSKE & $5 \%$ & $251 \mathrm{t}$ & $\begin{array}{c}0.47 \\
(0.98)\end{array}$ & $\begin{array}{c}0.47 \\
(0.98)\end{array}$ & $\begin{array}{c}0.33 \\
(0.91)\end{array}$ & $\begin{array}{c}0.53 \\
(1.02)\end{array}$ & $\begin{array}{c}0.60 \\
(1.05)\end{array}$ & $\begin{array}{l}0.48 \\
0.99\end{array}$ & 56.36 \\
\hline $\mathrm{T} 4$ & $\begin{array}{l}\text { Karanj seed } \\
\text { extract }\end{array}$ & $2.5 \%$ & $12.51 \mathrm{t}$ & $\begin{array}{c}0.53 \\
(1.01)\end{array}$ & $\begin{array}{c}0.53 \\
(1.01)\end{array}$ & $\begin{array}{c}0.47 \\
(0.98)\end{array}$ & $\begin{array}{c}0.60 \\
(1.05)\end{array}$ & $\begin{array}{c}0.67 \\
(1.08)\end{array}$ & $\begin{array}{c}0.57 \\
(1.03)\end{array}$ & 48.18 \\
\hline T 5 & $\begin{array}{l}\text { Garlic+green } \\
\text { chilli }\end{array}$ & $8.75 \mathrm{~kg}$ & $8.75 \mathrm{~kg}$ & $\begin{array}{c}0.53 \\
(1.01)\end{array}$ & $\begin{array}{c}0.40 \\
(0.95)\end{array}$ & $\begin{array}{c}0.33 \\
(0.91)\end{array}$ & $\begin{array}{c}0.47 \\
(0.98)\end{array}$ & $\begin{array}{c}0.53 \\
(1.02)\end{array}$ & $\begin{array}{c}0.43 \\
(0.96)\end{array}$ & 60.90 \\
\hline T 6 & Green chilli & $9 \mathrm{~kg}$ & $9 \mathrm{~kg}$ & $\begin{array}{c}0.47 \\
(0.98)\end{array}$ & $\begin{array}{c}0.53 \\
(1.02)\end{array}$ & $\begin{array}{c}0.53 \\
(1.02)\end{array}$ & $\begin{array}{c}0.60 \\
(1.05)\end{array}$ & $\begin{array}{c}0.73 \\
(1.11)\end{array}$ & $\begin{array}{c}0.60 \\
(1.05)\end{array}$ & 45.45 \\
\hline $\mathrm{T} 7$ & Red chilli & $9 \mathrm{~kg}$ & $9 \mathrm{~kg}$ & $\begin{array}{c}0.60 \\
(1.05)\end{array}$ & $\begin{array}{c}0.60 \\
(1.05)\end{array}$ & $\begin{array}{c}0.60 \\
(1.05)\end{array}$ & $\begin{array}{c}0.80 \\
(1.14)\end{array}$ & $\begin{array}{c}0.87 \\
(1.17)\end{array}$ & $\begin{array}{c}0.72 \\
(1.10)\end{array}$ & 34.54 \\
\hline $\mathrm{T} 8$ & $\begin{array}{l}\text { Triazophos } \\
40 \text { EC }\end{array}$ & $750 \mathrm{ml}$ & $750 \mathrm{ml}$ & $\begin{array}{c}0.40 \\
(0.95)\end{array}$ & $\begin{array}{c}0.33 \\
(0.91)\end{array}$ & $\begin{array}{c}0.27 \\
(0.87)\end{array}$ & $\begin{array}{c}0.40 \\
(0.95)\end{array}$ & $\begin{array}{c}0.47 \\
(0.98)\end{array}$ & $\begin{array}{c}0.37 \\
(0.93)\end{array}$ & 66.36 \\
\hline Т 9 & Control & - & - & $\begin{array}{c}0.73 \\
(1.11)\end{array}$ & $\begin{array}{c}1.00 \\
(1.22)\end{array}$ & $\begin{array}{c}1.07 \\
(1.25)\end{array}$ & $\begin{array}{c}1.13 \\
(1.28)\end{array}$ & $\begin{array}{c}1.20 \\
(1.30)\end{array}$ & $\begin{array}{c}1.10 \\
(1.26)\end{array}$ & - \\
\hline & C.D. at $5 \%$ & & & $\mathrm{NS}$ & 0.11 & 0.10 & 0.06 & 0.08 & - & - \\
\hline
\end{tabular}


Table.3 Efficacy of plant products against semilopper (C. acuta) in soybean during Kharif, 2015 after first spray

\begin{tabular}{|c|c|c|c|c|c|c|c|c|c|c|}
\hline \multicolumn{11}{|c|}{ Mean population of semilopper per/mrl } \\
\hline & & \multicolumn{8}{|c|}{ Post treatment observation } & \multirow{2}{*}{$\begin{array}{c}\text { Percent } \\
\text { reduction of } \\
\text { insect population } \\
\text { over control }\end{array}$} \\
\hline & Treatments & Dose & $\begin{array}{c}\text { Quantit } \\
\text { y /ha }\end{array}$ & $\begin{array}{l}\text { Pre treat. } \\
\text { Obs. }\end{array}$ & 1 DAS & 3 DAS & 7 DAS & 15 DAS & Mean & \\
\hline $\mathrm{T} 1$ & Neem oil & $2 \%$ & $101 \mathrm{t}$ & $\begin{array}{c}1 \\
(1.05)^{*}\end{array}$ & $\begin{array}{c}0.73 \\
(1.11) \\
\end{array}$ & $\begin{array}{c}0.67 \\
(1.08) \\
\end{array}$ & $\begin{array}{c}0.87 \\
(1.17) \\
\end{array}$ & $\begin{array}{c}0.67 \\
(1.08) \\
\end{array}$ & $\begin{array}{c}0.73 \\
(1.11) \\
\end{array}$ & 38.13 \\
\hline $\mathrm{T} 2$ & Karanj oil & $2 \%$ & $101 \mathrm{t}$ & $\begin{array}{c}1.07 \\
(1.08) \\
\end{array}$ & $\begin{array}{c}0.67 \\
(1.08) \\
\end{array}$ & $\begin{array}{c}0.73 \\
(1.11) \\
\end{array}$ & $\begin{array}{c}0.80 \\
(1.14) \\
\end{array}$ & $\begin{array}{c}0.53 \\
(1.02) \\
\end{array}$ & $\begin{array}{c}0.68 \\
(1.09) \\
\end{array}$ & 42.37 \\
\hline T 3 & NSKE & $5 \%$ & $251 \mathrm{t}$ & $\begin{array}{c}0.93 \\
(1.05) \\
\end{array}$ & $\begin{array}{c}0.53 \\
(1.02) \\
\end{array}$ & $\begin{array}{c}0.60 \\
(1.05) \\
\end{array}$ & $\begin{array}{c}0.67 \\
(1.08) \\
\end{array}$ & $\begin{array}{c}0.33 \\
(0.91) \\
\end{array}$ & $\begin{array}{c}0.53 \\
(1.01) \\
\end{array}$ & 55.08 \\
\hline $\mathrm{T} 4$ & $\begin{array}{l}\text { Karanj seed } \\
\text { extract }\end{array}$ & $2.5 \%$ & $12.51 \mathrm{t}$ & $\begin{array}{c}1 \\
(1.10)\end{array}$ & $\begin{array}{c}0.60 \\
(1.05) \\
\end{array}$ & $\begin{array}{c}0.67 \\
(1.08)\end{array}$ & $\begin{array}{c}0.73 \\
(1.11) \\
\end{array}$ & $\begin{array}{c}0.60 \\
(1.05)\end{array}$ & $\begin{array}{c}0.65 \\
(1.07)\end{array}$ & 44.91 \\
\hline $\mathrm{T} 5$ & $\begin{array}{l}\text { Garlic+green } \\
\text { chilli }\end{array}$ & $8.75 \mathrm{~kg}$ & $8.75 \mathrm{~kg}$ & $\begin{array}{c}0.87 \\
(105)\end{array}$ & $\begin{array}{c}0.47 \\
(0.98) \\
\end{array}$ & $\begin{array}{c}0.53 \\
(1.02) \\
\end{array}$ & $\begin{array}{c}0.60 \\
(1.05) \\
\end{array}$ & $\begin{array}{c}0.47 \\
(0.98) \\
\end{array}$ & $\begin{array}{c}0.52 \\
(1.01) \\
\end{array}$ & 55.93 \\
\hline Т 6 & Green chilli & $9 \mathrm{~kg}$ & $9 \mathrm{~kg}$ & $\begin{array}{c}1.13 \\
(1.10) \\
\end{array}$ & $\begin{array}{c}0.67 \\
(1.08) \\
\end{array}$ & $\begin{array}{c}0.80 \\
(1.14) \\
\end{array}$ & $\begin{array}{c}0.87 \\
(1.17) \\
\end{array}$ & $\begin{array}{c}0.73 \\
(1.11) \\
\end{array}$ & $\begin{array}{c}0.77 \\
(1.12) \\
\end{array}$ & 34.75 \\
\hline $\mathrm{T} 7$ & Red chilli & $9 \mathrm{~kg}$ & $9 \mathrm{~kg}$ & $\begin{array}{c}1.13 \\
(1.10) \\
\end{array}$ & $\begin{array}{c}0.87 \\
(1.17) \\
\end{array}$ & $\begin{array}{c}0.87 \\
(1.17) \\
\end{array}$ & $\begin{array}{c}0.93 \\
(1.19) \\
\end{array}$ & $\begin{array}{c}0.80 \\
(1.14) \\
\end{array}$ & $\begin{array}{c}0.87 \\
(1.17) \\
\end{array}$ & 26.27 \\
\hline T 8 & $\begin{array}{l}\text { Triazophos } \\
40 \text { EC }\end{array}$ & $750 \mathrm{ml}$ & $750 \mathrm{ml}$ & $\begin{array}{c}0.80 \\
(1.17)\end{array}$ & $\begin{array}{c}0.40 \\
(0.94)\end{array}$ & $\begin{array}{c}0.40 \\
(0.95)\end{array}$ & $\begin{array}{c}0.47 \\
(0.98)\end{array}$ & $\begin{array}{c}0.33 \\
(0.90)\end{array}$ & $\begin{array}{c}0.40 \\
(0.94)\end{array}$ & 66.10 \\
\hline T 9 & Control & - & - & $\begin{array}{c}1.20 \\
(1.30) \\
\end{array}$ & $\begin{array}{c}0.93 \\
(1.20) \\
\end{array}$ & $\begin{array}{c}1.20 \\
(1.30) \\
\end{array}$ & $\begin{array}{c}1.27 \\
(1.33) \\
\end{array}$ & $\begin{array}{c}1.33 \\
(1.35) \\
\end{array}$ & $\begin{array}{c}1.18 \\
(1.30) \\
\end{array}$ & - \\
\hline & C.D. at $5 \%$ & & & NS & 0.10 & 0.08 & 0.09 & 0.15 & - & - \\
\hline
\end{tabular}


Table.4 Efficacy of plant products against semilopper (C. acuta) in soybean during Kharif, 2015 after second spray

\begin{tabular}{|c|c|c|c|c|c|c|c|c|c|c|}
\hline \multicolumn{11}{|c|}{ Mean population of semilopper per mrl } \\
\hline & & \multicolumn{8}{|c|}{ Post treatment observation } & \multirow{2}{*}{$\begin{array}{c}\text { percent } \\
\text { reduction of } \\
\text { insect population } \\
\text { over control }\end{array}$} \\
\hline & Treatments & Dose & $\begin{array}{c}\text { Quantit } \\
\text { y /ha }\end{array}$ & $\begin{array}{l}\text { Pre treat. } \\
\text { Obser. }\end{array}$ & 1 DAS & 3 DAS & 7 DAS & 15 DAS & Mean & \\
\hline $\mathrm{T} 1$ & Neem oil & $2 \%$ & $101 \mathrm{t}$ & $\begin{array}{c}0.73 \\
(1.11)^{*}\end{array}$ & $\begin{array}{c}0.67 \\
(1.08)\end{array}$ & $\begin{array}{c}0.60 \\
(1.05)\end{array}$ & $\begin{array}{c}0.53 \\
(1.02)\end{array}$ & $\begin{array}{c}0.73 \\
(1.11)\end{array}$ & $\begin{array}{c}0.63 \\
(1.06)\end{array}$ & 42.72 \\
\hline T 2 & Karanj oil & $2 \%$ & $101 \mathrm{t}$ & $\begin{array}{c}0.80 \\
(1.14)\end{array}$ & $\begin{array}{c}0.60 \\
(1.05) \\
\end{array}$ & $\begin{array}{c}0.53 \\
(1.02) \\
\end{array}$ & $\begin{array}{c}0.47 \\
(0.98) \\
\end{array}$ & $\begin{array}{c}0.67 \\
(1.05) \\
\end{array}$ & $\begin{array}{c}0.57 \\
(1.03)\end{array}$ & 48.18 \\
\hline T 3 & NSKE & $5 \%$ & $251 \mathrm{t}$ & $\begin{array}{c}0.67 \\
(1.05) \\
\end{array}$ & $\begin{array}{c}0.47 \\
(0.98) \\
\end{array}$ & $\begin{array}{c}0.40 \\
(0.94) \\
\end{array}$ & $\begin{array}{c}0.33 \\
(0.91) \\
\end{array}$ & $\begin{array}{c}0.53 \\
(1.02) \\
\end{array}$ & $\begin{array}{c}0.43 \\
(0.96) \\
\end{array}$ & 60.90 \\
\hline $\mathrm{T} 4$ & $\begin{array}{l}\text { Karanj seed } \\
\text { extract }\end{array}$ & $2.5 \%$ & $12.51 \mathrm{t}$ & $\begin{array}{c}0.73 \\
(1.11)\end{array}$ & $\begin{array}{c}0.53 \\
(1.02)\end{array}$ & $\begin{array}{c}0.47 \\
(0.98)\end{array}$ & $\begin{array}{c}0.40 \\
(0.94)\end{array}$ & $\begin{array}{c}0.60 \\
(1.05)\end{array}$ & $\begin{array}{c}0.50 \\
(1.00)\end{array}$ & 54.54 \\
\hline T 5 & $\begin{array}{l}\text { Garlic+green } \\
\text { chilli }\end{array}$ & $8.75 \mathrm{~kg}$ & $8.75 \mathrm{~kg}$ & $\begin{array}{c}0.60 \\
(1.05)\end{array}$ & $\begin{array}{c}0.40 \\
(0.94)\end{array}$ & $\begin{array}{c}0.33 \\
(0.91)\end{array}$ & $\begin{array}{c}0.27 \\
(0.87)\end{array}$ & $\begin{array}{c}0.47 \\
(0.98)\end{array}$ & $\begin{array}{c}0.37 \\
(0.93)\end{array}$ & 66.36 \\
\hline Т 6 & Green chilli & $9 \mathrm{~kg}$ & $9 \mathrm{~kg}$ & $\begin{array}{c}0.87 \\
(1.17) \\
\end{array}$ & $\begin{array}{c}0.60 \\
(1.05)\end{array}$ & $\begin{array}{c}0.53 \\
(1.02) \\
\end{array}$ & $\begin{array}{c}0.60 \\
(1.05)\end{array}$ & $\begin{array}{c}0.73 \\
(1.11)\end{array}$ & $\begin{array}{c}0.62 \\
(1.06) \\
\end{array}$ & 43.64 \\
\hline $\mathrm{T} 7$ & Red chilli & $9 \mathrm{~kg}$ & $9 \mathrm{~kg}$ & $\begin{array}{c}0.93 \\
(1.20)\end{array}$ & $\begin{array}{c}0.80 \\
(1.14) \\
\end{array}$ & $\begin{array}{c}0.73 \\
(1.11) \\
\end{array}$ & $\begin{array}{c}0.67 \\
(1.05)\end{array}$ & $\begin{array}{c}0.87 \\
(1.17)\end{array}$ & $\begin{array}{c}0.77 \\
(1.12)\end{array}$ & 30.00 \\
\hline T 8 & $\begin{array}{l}\text { Triazophos } \\
40 \text { EC }\end{array}$ & $750 \mathrm{ml}$ & $750 \mathrm{ml}$ & $\begin{array}{c}0.53 \\
(1.02) \\
\end{array}$ & $\begin{array}{c}0.33 \\
(0.91) \\
\end{array}$ & $\begin{array}{c}0.27 \\
(0.87) \\
\end{array}$ & $\begin{array}{c}0.20 \\
(0.83) \\
\end{array}$ & $\begin{array}{c}0.33 \\
(0.91) \\
\end{array}$ & $\begin{array}{c}0.28 \\
(0.88) \\
\end{array}$ & 74.54 \\
\hline Т 9 & Control & - & - & $\begin{array}{c}1.13 \\
(1.28) \\
\end{array}$ & $\begin{array}{c}1.07 \\
(1.25) \\
\end{array}$ & $\begin{array}{c}1.13 \\
(1.28) \\
\end{array}$ & $\begin{array}{c}1.07 \\
(1.25) \\
\end{array}$ & $\begin{array}{c}1.13 \\
(1.28) \\
\end{array}$ & $\begin{array}{c}1.10 \\
(1.26)\end{array}$ & - \\
\hline & C.D. at $5 \%$ & & & NS & 0.10 & 0.13 & 0.22 & 0.18 & - & - \\
\hline
\end{tabular}


Table.5 Assessment of benefit cost ratio in soybean Kharif, 2015

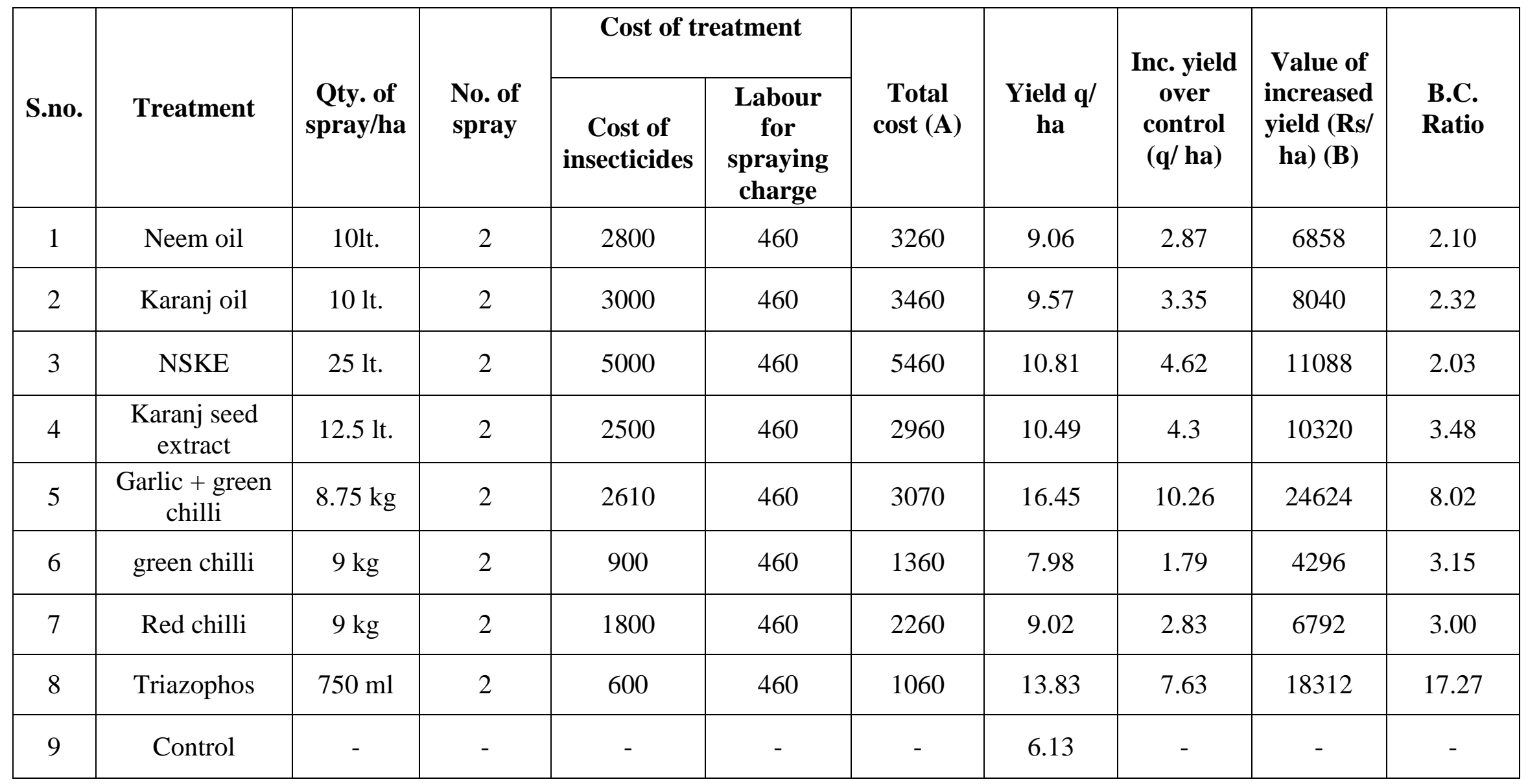


Percent reduction of $S$. litura population over control after second spray

Percent reduction was higher in triazophos @ $750 \mathrm{ml} / \mathrm{ha}(66.36 \%)$ treated crop. Among the plant products, garlic+green chilli $8.75 \mathrm{~kg} / \mathrm{ha}$ $(60.40 \%)$ followed by NSKE @ 5\% $(56.36 \%)$, Karanj seed extract @ 2.5\% (48.18\%), Karanj oil @ 2\% (47.27\%), Neem oil @ 2\% (47.27\%), green chilli @ 9kg/ha (45.45\%).It was lowest in red chilli @ 9kg/ha treated plots and recorded only 34.54 percent reduction in insect population.

\section{Overall mean population of $C$. acuta after first spray}

Mean larval population during first spray indicated that chemical insecticide i.e. triazophos40 EC @ 750 ml/ha recorded the minimum population of 0.40 larva/mrl and among the different plant products, garlic+green chilli@8.75 kg/ha recorded minimum larval population with 0.52 larva/mrl, followed by NSKE @ 5\% (0.53), Karanj seed extract @ 2.5\% (0.65), Karanj oil @ 2\% (0.68), Neem oil @ 2\% (0.73), green chilli@9kg/ha (0.77 larva/mrl) and it was maximum in red chilli @ 9kg/ha having 0.87 larva/mrl.

\section{Percent reduction of $C$. acuta population over control after first spray}

Percent reduction was higher in triazophos @ $750 \mathrm{ml} / \mathrm{ha}(66.10 \%)$ treated crop and among the plant products, it was maximum in garlic+green chilli @ $8.75 \mathrm{~kg} / \mathrm{ha}(55.93 \%)$ treated crop followed by NSKE @ 5\% (55.08\%), Karanj seed extract @ 2.5\% (44.91\%), Karanj oil @ 2\% (42.37\%), Neem oil @ 2\% (38.13\%), green chilli @ 9kg/ha (34.75\%) and minimum of 26.27 percent recorded in red chilli@9kg/ha treated plots.

Over all mean population of $C$. acuta after second spray: Mean larval population during second spray indicated that chemical insecticide i.e. triazophos @ $750 \mathrm{ml} / \mathrm{ha}$ recorded the minimum population of 0.28 larva/mrl and among the different plant products, garlic+green chilli @ 8.75 kg/ha recorded minimum larval population with 0.37larva/mrl followed by NSKE @ 5\% (0.47), Karanj seed extract @ 2.5\% (0.57), Karanj oil @2\% (0.58), Neem oil @ 2\% (0.58), green chilli @ 9kg/ha (0.60) and it was maximum in red chilli@9kg/ha having 0.72 larva/mrl.

Percent reduction of $C$. acuta population over control after second spray

Percent reduction higher in triazophos 40 EC @ $750 \mathrm{ml} / \mathrm{ha}(74.54 \%)$ treated crop, and among the plant products, it was maximum in garlic+green chilli@8.75 kg/ha $(66.36 \%)$ treated crop followed by NSKE @ 5\% (60.90\%), Karanj seed extract @ 2.5\% (54.54\%), Karanj oil @ 2\% (48.18\%), green chilli @ 9kg/ha (43.64\%), Neem oil @ 2\% $(42.72 \%)$ and lowest in red chilli @ 9kg/ha treated plots was recorded only 43.64 percent reduction in insect population.

The results are in conformity with the findings of Vijayalakshmi et al., (1997) who reported that ginger extract as natural pesticide, alone and in combination with other plant products like chilli, garlic and cow urine as effective plant products against $H$. armigera.

Lakshmanan (2001) also reported that the garlic bulb extract alone or in combination with other plant extracts were effective in managing the several lepidopteran pests viz., Earias vitella, Chilo partellus (Swinhoe), Corcyra cephalonica Staint., Helicoverpa armigera and Spodoptera litura. Choudhary and Shrivastava (2007) reported that application of neem seed kernel extract (NSKE) at 5\% + neem leaf extract (NLE) at $10 \%$ reduced the maximum larval population. 


\section{Assessment of benefit cost ratio}

Among the plant products, the maximum benefit cost ratio was found in the treatment garlic+green chilli@8.75 kg/ha having 8.02. In rest of the plant products, more or less similar benefit cost ratio was obtained like 3.48 in Karanj seed extract @ 2.5\% which was followed by green chilli @ 9kg/ha (3.15), red chilli@9kg/ha (3.00), Karanj oil @ 2\% (2.32), Neem oil @ 2\% (2.10) and the minimum benefit cost ratio was recorded in the treatment NSKE @ 5\% having only 2.03 and among the chemical treatment triazophos @ 750ml/ha which was used for management of insect pests of soybean the benefit cost ratio was maximum with 17.27.

Present findings are in agreement with those of Raghuvanshi et al., (2014) as they reported that triazophos gave the maximum better return on soybean crop. In contradictory Panchabhavi et al., (1994) reported that lower pod damage and higher seed yield were recorded on pigeon pea when fenvalerate was applied twice at 15 days interval. However, highest cost benefit ratio was obtained in NSKE sprayed at 15 days interval with a seed yield of $12.0 \mathrm{q} / \mathrm{ha}$.

\section{References}

Asoken, R., Mohandas, S. and Anand. 2000. Biofertilizer and biopesticides for horticultural crop. Indian Hort., 44-47.
Choudhary, A.K. and Shrivastava, S.K. 2007. Efficacy and economics of some neem based products against tobacco caterpillar, Spodoptera litura F. on soybean in Madhya Pradesh, India. Int. J. Agri. Sci., 3(2): 15-17.

Isman, M.B. 2006. Botanical insecticides, deterrents and repellents in modern agriculture and increasingly regulated world. Ann. Rev. Entomol., 51: 45-66.

Lakshmanan, K.K. 2001. Garlic a natural pesticide. The Hindu, March, 1, p. 8 .

Panchabhavi, K.S., Kotikal, Y.K., Krishna Naik, L. Giraddi, R. S., and Yelshetty, S., 1994, A note on efficacy of sequential spray of neem seed extract and insecticides for the control of pod borer $\mathrm{H}$. armigera infesting redgram (Cajanus cajana (L) Mill sp.). Karnataka $J$. Agric. Sci., 7: 353-357.

Prakash, A. and Rao, J. 1989. Begunia leaves: a pulse grain protectant. Indian J. Entomol., 51(2):192-195.

Prakash, A. Rao. and Jagadiswari. 2003. Management of rice storage incest. CRRI, Tech. Bull., 17: 4 PP.

Raghuvanshi, S., Bhadauria, N.S. and Singh, P. 2014. Efficacy of Insecticides against Major Insect Pests of Soybean [Glycine max (L.)Merrill]. Trends in Biosci., 7(3): 191- 193.

Vijayalakshmi, K. Subhashini, B. and Shivani, V. K. 1996. Plants in pest Control Garlic and Onion, Centre for Indian Knowledge Systems, Chennai, pp. $1-20$.

\section{How to cite this article:}

Toshima Kushram, Y.K. Yadu, M.K. Sahu, A.K. Kulmitra and Kumar, R. 2017. Bio Efficacy of Botanical Insecticides against Defoliators Pests on Soybean. Int.J.Curr.Microbiol.App.Sci. 6(3): 2196-2204. doi: https://doi.org/10.20546/ijcmas.2017.603.250 